\title{
Using a storytelling intervention in schools to explore death, dying, and loss.
}

\begin{abstract}
Purpose: Experiencing bereavement in childhood can cause profound changes to developmental trajectories. This study evaluated the feasibility of implementing a public health intervention in schools to encourage pupils aged 12-15 years to independently explore ideas of death, dying, loss, and end of life care in a structured and creative format.
\end{abstract}

Design: A co-produced storytelling intervention was implemented in an independent school in Norwich, UK. Pupils wrote up to 1000 words in response to the title, "I Wish We'd Spoken Earlier". Their participation was voluntary and extra-curricular. Stakeholder feedback was used in addition to the submissions as a measure of acceptability, appropriateness, adoption, and feasibility.

Findings: 24 entries were submitted. Pupils demonstrated their ability to engage thoughtfully and creatively with the subject matter. Feasibility for the storytelling intervention was demonstrated. Importantly, the intervention also prompted family conversations around preferences and wishes for end of life care.

Implications: Educational settings can be considered as anchor institutions to support a public health approach to end of life care.

Research limitations / implications: To determine whether the intervention has psychological and social benefits will require further study.

Originality / value: The positive response from all stakeholders in delivering and supporting the intervention indicates that schools are a community asset that could be further empowered to support children and families affected by death, dying, and loss.

Keywords: public health, storytelling, children, death, dying, bereavement, end of life care

Article Classification: Research paper

\section{Introduction}

Death is often a source of fear and anxiety and is generally avoided as a topic of conversation (Kellehear, 2014). In the UK, 1 in 29 children (approximately one child in every class) will be bereaved of a parent or sibling, while 1 in 16 will be bereaved of a close friend (Penny and Stubbs, 2015; Winston's Wish, 2020). These children are at increased risk of poorer mental and physical health, lower educational attainment, substance misuse, criminal activity, and early mortality (Guldin et al., 2015; Kaplow et al., 2010; Stikkelbroek et al., 2016). Bereavement in childhood may therefore disrupt the trajectory expected in healthy development. Compassionate 
Communities, a public health approach to palliative and end of life care, aims to reduce the impact of these negative physical, social, psychological, and spiritual outcomes by improving community participation and local support networks. (Wegleitner et al., 2015).

The public health approach to palliative and end of life care recognises that experiences of death, dying, loss, and end of life care are universal and that there is a need for enhanced peer-support, and shared responsibility within communities (Abel and Kellehear, 2016). In Norfolk and Waveney, the region where the intervention took place, 'Each community is prepared to help' is one of six ambitions within the regional 'Palliative and End of Life Care Strategy', which aligns with national end of life care guidelines. (The Norfolk and Waveney Health and Care Partnership, 2020; The National Council for Palliative Care, 2016). The public health approach identifies schools as valuable sources of support and guidance (Sallnow and Paul, 2018), and key in implementing sustainable social change (Compassionate Communities, 2020).

Death education and peer-support can facilitate knowledge sharing, promote mental wellbeing, and reduce stigma and social isolation that can be associated with death, loss, or caregiving (Kennedy et al., 2017). These methods align with principles of 'positive education', which aims to promote children's mental wellbeing and resilience, therefore optimising their development and enabling them to flourish (Norrish et al., 2013). However, some teachers report that they do not feel comfortable discussing death and dying with their pupils, and that they feel illequipped to manage a death in the school community (Child Bereavement UK, 2018; Galende, 2015). Feedback from pupils also suggests that support in this context is often lacking (McGovern and Tracey, 2010). This suggests that school communities may benefit from additional support in developing their capacity and partnership role as anchor institutions in the provision of community-based end of life care.

\section{Asset-Based Community Development (ABCD)}

$A B C D$ is a well-developed approach to sustainable community-driven development (Kretzman and McKnight, 1993). Its premise is that communities can respond collectively by mobilising existing, but often unrecognised assets. Schools have previously been critiqued for being disconnected from local communities (Kretzman and McKnight, 1993). However, their potential to share a partnership role has also been recognised (Caan, 2015), and initiatives to integrate health promotion in schools have been developed internationally (Forrester, 2018; Jensen et al., 2017). These initiatives have been effective in improving a range of health outcomes including; physical activity, diet, and reducing bullying and tobacco use (Langford et al., 2014). A school-level ABCD approach that aims to counteract inequalities in child development has been advocated (Caan, 2015). However, the feasibility of implementing a structured creative intervention to offer school-aged children the opportunity to explore their ideas around death, dying, loss, and care, and to encourage more conversations about end of life requires further consideration. 
The Compassionate Cities Charter recommends storytelling competitions as one way to raise awareness of death and dying (Compassionate Communities, 2020). Storytelling has been used in communities across the world for centuries to express, appreciate, and understand the ubiquitous nature of grief. Recent research demonstrates the positive impact of storytelling interventions on mental health and wellbeing for adults and children alike (Gentle et al., 2020; Gillam 2018). It does not require specialist knowledge or equipment, and is therefore a highly accessible medium for both tellers and listeners (McCall et al., 2019; Scott et al., 2013). Storytelling can help individuals and families gain a sense of control over events, relieve emotional tension, make meaning out of experiences, and connect different people's experiences (Bosticco and Thompson, 2005). This is echoed by Kellehear (2014) and Rumbold et al. (2011) who describe storytelling as a cultural resource for communities that can be used to facilitate a sense of predictability, normalise an experience, increase connectedness, and reduce isolation. Glazer and Marcum (2011) describe the use of storytelling to assist children who have experienced the death of a family member or friend, showing that children are able to use narrative to express grief and loss, and that artwork can aid in the expression of both experiences. The evidence base therefore suggests that supporting children to use storytelling in a structured environment as a medium to explore death and dying may have beneficial health and social outcomes and could therefore be an effective method to integrate into the public health approach to end of life care. However, there is also a need for sensitivity due to the complex nature of the topic (Ferow, 2019; LaFreniere and Cain, 2015; van der Geest et al., 2015). The purpose of this study was pragmatic, rather than theory-driven (Smith, 2011).

\section{Specific Aims}

- To implement an intervention that adopts a public health approach to palliative and end of life care.

- To determine whether the intervention is acceptable to and appropriate for the school community.

- To explore whether using storytelling to facilitate the exploration of death and dying could feasibly be incorporated into the school curriculum.

- To explore the extent to which the project was adopted within the school and local community

\section{Method}

\section{Context}

The intervention was conducted at Norwich School, an independent school in Norwich, UK for children aged four to 18. The school has a long tradition of involvement in the arts and civic engagement within its outreach programmes.

\section{Intervention}

Pupils were invited to produce a piece of writing as a response to the prompt "I Wish We'd Spoken Earlier", a phrase used by Dying Matters, a UK campaign that aims to 
enable people to talk more openly about death (Dying Matters, 2019). Participation was voluntary. The maximum word count for entries was 1000 words.

In line with the public health approach, the project design was co-produced between Norwich School, Norfolk and Waveney NHS Palliative and End of Life Care Collaborative (The Norfolk and Waveney Health and Care Partnership, 2020), Dying Matters, and the University of East Anglia.

In autumn 2018, pupils in years 8, 9 and 10 (aged 12-15 years), a cohort of approximately 300 , were invited to participate in a creative writing project. Schools in the local area were also invited to take part. Pupils were asked to produce a piece of writing as part of their holiday homework, to support their literacy, and Personal Social, Health, and Economic (PSHE) educational programmes. As well as the initial launch of the creative writing project at a school assembly, supporting material developed by Norwich School and Dying Matters, including suggestions and prompts, were provided to assist pupils, parents, and teachers with the project.

Invitations and guidelines were posted on the Norwich School and Dying Matters websites; a news story about the project went on the school website, and a feature was published on their outreach webpage. An email press release was sent out to schools in the region, and schools with whom they are closely affiliated were contacted. A dedicated school email address was created to receive submissions.

\section{Implementation Measures}

The project utilised four implementation measures as described by Proctor et al. (2011): acceptability, appropriateness, adoption, and feasibility. Due to the project's scale, and the difficulty in evaluating the 'dynamic cascading process' of community engagement (Kumar, 2020), quantitative outcome measures were not incorporated into the study design. To gauge acceptability and appropriateness, feedback from pupils and parents, an appraisal from the school, and the perceived quality of engagement evident in the submissions were evaluated. Wider support from the local community and other schools was used as a measure of adoption. Feasibility of implementation was gauged by the extent to which all stakeholders believed the intervention had been applied successfully.

\section{Ethical Considerations}

The project was led by the school with assistance from UEA and Dying Matters and was therefore subject to the school's existing governance and policies around safeguarding and pastoral support. This was important, considering the potentially emotive nature of the subject matter. Staff from all specialities as well as nonteaching personnel were aware of the project, and parents of the participating pupils consented to their involvement.

Written consent was obtained from the school and the report was approved prior to dissemination. Written consent was also obtained from the parents of three winners to include them as co-authors. Individual names and identifying information given in the feedback have been changed and marked with an asterisk $\left(^{*}\right)$. The submissions in the appendix have also been anonymised. 


\section{Results}

\section{Entries}

There were 24 entries from Norwich School pupils. These included poetry, song lyrics, and prose. The content included descriptions of a death in the family, selfharm, catastrophic injury, and suicide. Some were seemingly fantasy, whilst others were anchored in real-life. All entries named, described, and reflected on the dying experience; painting pictures of how it feels to be dying, dead, bereaved, or losing someone. A panel of three judges appraised all 24 entries. The panel comprised two academics from the University of East Anglia, who acted as independent judges, and a further judge from Norwich School. Three entries were agreed by consensus as most impactful in terms of the writers' level of engagement with the subject matter and the expression of their ideas (appendix). The winning pupils were invited to read their submissions to an audience of approximately 100 people including; medics, care professionals, charity members, and industry professionals at the local Dying Matters conference. They received a standing ovation for their work. All 24 stories were compiled in a booklet and displayed as part of a creative writing exhibition at the school.

\section{Feedback and Reflections}

Feedback was collected as free commentary from participants and attendees at the Dying Matters conference. Pupils who had submitted one of the 24 entries and their parents were also asked to provide written reflections on the project when invited to consent for their work to be included in the compilation. Examples of the written reflections are reported below:

- Pupil authors

"Taking part in the Dying Matters project was at first just another poetry competition. However, when we spoke at the conference it brought a new perspective on what death meant to others and how important it is to talk about it. After the conference I started talking to my parents about how we can help them when they get older as a family. We also discussed our wishes for after each of our deaths including mine if I die before them. It has been such an interesting project that has explored a topic that most people vehemently avoid."

"I did struggle to talk or write about death, and it took me multiple attempts to write a piece that I was proud of and felt connected to. I had recently lost a family member and was determined to not use my own experience in my writing, which meant that I never engaged with what I was doing. I eventually realised that writing about what I knew was the only way I was going to write a piece I was happy with, instead of getting frustrated with myself. Since writing the piece and reading it at the conference, I feel much more open to talking about my experience and, although I don't do it all the time, it's a comfort to know that I am able to."

- Parents

"Death is never an easy subject matter at the best of times and even more sensitive when trying to talk to young people about it. $D^{*}$ voluntarily decided to contribute to Dying Matters to reflect on this theme. My own father had died very suddenly when $D^{*}$ was a toddler. We had spoken about this as a family and I had expressed my sincere regret about not having had 
those grown up conversations with my own father during his lifetime. These discussions formed the basis of D's* contribution. Since writing their poem my youngest sister has sadly died aged just 39*. As a family we made sure that her achievements and our love for her were duly celebrated in her lifetime and that she knew just how much she meant to us while still around to hear it - by talking about death we commemorated her life".

"The first I was aware of $E^{*}$ writing a poem for the Dying Matters Writing Project was when $E^{*}$ sat at the piano and used music to write what they were thinking. They didn't speak with me very much during this part of the process, but when the poem was finished they shared it with me. I was really struck by how much emotion and depth went into the poem. Afterwards, we didn't talk a lot about the poem itself, but it catalysed conversations between the two of us about what they would like to happen after they died if they died before us and also about what we would like in our old age. It was a really interesting and worthwhile process"

"The Dying Matters project has been very interesting for my family. It provoked dialogue amongst us and offered opportunity to reflect on our feelings and enabled us to share thoughts. This was not, by any means, easy - particularly after recently losing a much-loved member of our family. I was very mindful of how the project might impact on us as we are still navigating our way through this loss and we did have one or two very poignant conversations whilst $F^{*}$ was working on their creative writing piece. Whilst the subject matter is painful, it is important to keep the conversation going and reflecting back on the project I do believe that it was beneficial for my family to have been part of. I don't think $F^{*}$ will mind me saying that for them this was a challenging remit, one which took them numerous attempts to complete. I was so proud of their determination to write something emotive but very different from their own experience and their final attempt was very moving. I was delighted for $F^{*}$ that they had the opportunity to read their piece at the conference. This meant a lot to them."

The pupils clearly demonstrated their ability to engage thoughtfully and creatively in diverse and individual ways during the intervention. The work showed pupils' deeper understanding of how death affects families. Importantly, pupils' engagement generated further discussions within families about death, dying, and loss, including reflections on past events and future wishes.

\section{Implementation Outcomes}

\section{- Acceptability and Appropriateness}

Norwich School supervised pupils in completing their written submissions, advertised the project in assemblies, designed and produced project-related web pages, and accompanied pupils in attending the Dying Matters conference. This indicates that the project was well-supported and deemed acceptable by school staff. Despite the challenging subject matter and extra-curricular nature of the activity, 24 entries were submitted. Three pupils presented their work at the conference and in assembly. Parents gave positive feedback and no adverse outcomes as a result of participating were reported. The pupils' submissions and testimonies were considerate and mature, suggesting that the use of storytelling to explore this topic was appropriate.

- Adoption 
The school appeared to fully embrace the project, as evidenced by their submissions and presence at the conference hosted in a community setting. However, despite inviting other schools to participate, no submissions from other schools were received. The process of inviting other schools was not evaluated, so the reason for this lack of wider adoption cannot be determined.

\section{- Feasibility}

Pupils from Norwich School produced high quality creative work, and were motivated and able to present their work both at the Dying Matters conference and in a whole school assembly. Although participation in the conference and assembly was voluntary, all agreed to present their work. They displayed a sense of ownership in developing a formal piece of creative writing. Norwich School supported the project throughout the process, and both pupils and parents gave positive feedback about the experience.

\section{Discussion}

The use of storytelling as a structured intervention to explore death, dying, and loss in schools demonstrated feasibility. Further exploration of ways to encourage wider participation is needed. There was a risk that exploring ideas surrounding the end of life would not stimulate interest, or potentially cause upset. However, the participatory nature of the storytelling exercise enabled active engagement and creative exploration. The intervention also appeared to prompt difficult but beneficial family conversations about recent bereavements, as well as conversations about end of life care preferences and wishes. In one report, involvement with the project appears to have had a positive influence on how a family responded to and supported a dying family member. By enabling these discussions, the intervention may have contributed to facilitating greater psychological preparation for and resilience to death, dying, and loss among families (Metzl et al., 2008), lessening the risk of negative developmental outcomes (Kaplow et al., 2010; Stikkelbroek et al., 2016), and complex bereavement (Wegleitner et al., 2015).

Whilst the project appeared feasible for children aged 12-15, it is unclear whether this intervention would be suitable for younger children. There is a breadth of children's fiction exploring concepts of death and loss suitable for ages $5-11$ (Child Bereavement UK, 2020). However, a paucity of research regarding its efficacy as a public health approach is apparent. Small group or school assembly interventions appropriate for primary school children have been developed. They explore concepts of loss in age-appropriate language (St Nicholas Hospice Care, 2020). The longer term outcomes of these health promoting interventions merit further study.

\section{Limitations}

Despite the success of the intervention, the project had a number of limitations. First, the project was unable to garner participation from other schools in the area. As no feedback was received from these schools it is difficult to attribute potential reasons. However, it could be speculated that some schools may lack some of the resources that enabled Norwich School to implement and oversee the project (Vijay and Monin, 
2018). Second, 24 entries from a possible cohort of 300 were submitted. Whilst the project was voluntary and set as extra-curricular holiday homework, it would be beneficial to further explore why more submissions were not received. Third, feedback was only sought from parents and pupils at the end of the project. Receiving feedback at different stages of the project, as well as feedback from teachers, and pupils who chose not to participate could have given insight into the separate processes involved in implementing the intervention. Fourth, as the project aimed to explore feasibility there was no evaluation of the intervention's effectiveness in embedding behavioural change, or its effect on mental wellbeing. However, the school has reported plans to embed the exercise within its curriculum suggesting longer term adoption of the intervention.

\section{Future Research}

Future research may wish to repeat the exercise at scale, and with a more rigorous analytical approach. This might include working with younger and older children. The study could evaluate outcomes using a socio-ecological framework to monitor change at individual, interpersonal, organisational, community, and policy levels (McLeroy et al., 1988). It may also be of benefit to determine the necessary resources schools need to participate in the project.

\section{Conclusion}

Whilst it is indicated elsewhere that teachers, pupils and parents frequently feel illequipped to engage in conversations around death and dying; using a creative storytelling intervention to approach the topic in schools appears to be an acceptable, appropriate and feasible approach. Moreover, feedback from parents and pupils suggested the intervention had prompted important and beneficial discussions about end of life care to take place in families, which may have otherwise been avoided. The positive response to the intervention indicates that schools are a valuable community asset that could be empowered to support children and families affected by death, dying, or loss.

\section{Funding}

Author one and two were supported with funding received from the Eastern Academic Health Science Network (EAHSN), UK. The views expressed are those of the authors, and not necessarily those of the EAHSN.

The corresponding author was supported by the UK National Institute of Health Research (NIHR) Applied Research Collaboration East of England (ARC EoE) programme. The views expressed are those of the authors, and not necessarily those of the NIHR, NHS or Department of Health and Social Care.

\section{REFERENCES}

Abel, J. and Kellehear, A. (2016), "Palliative care reimagined: a needed shift", BMJ Supportive and Palliative Care, Vol. 6, pp. 21-26. 
Abel J., Kellehear A., and Karapliagou, A. (2018), "Palliative care-the new essentials", Annals of Palliative Medicine (Public Health Approaches to Palliative Care), Vol. 7, S2, pp. S3-S14.

Bosticco, C. and Thompson, T. (2005), "Narratives and storytelling in coping with grief and bereavement", OMEGA - Journal of Death and Dying, Vol. 51, No.1, pp. 116.

Caan, W., Cassidy, J., Coverdale, G., Ha, M-A., Nicholson, W., and Rao, M. (2015), "The value of using schools as community assets for health", Public Health, Vol. 129, pp. 3-16.

Child Bereavement UK (2018), "Summary Research report: Improving bereavement support in schools", available at:

https://www.childbereavementuk.org/Handlers/Download.ashx?IDMF=fa7a443b636d-4238-af12-accedec84419 (accessed 7 April 2020).

Compassionate Communities (2020), "The Compassionate City Charter", available at: https://www.compassionate-communitiesuk.co.uk/the-compassionate-city-charter (accessed 27 May 2020).

Dying Matters (2019), "I wish we'd spoken earlier", available at: https://www.dyingmatters.org/blog/i-wish-wed-spoken-earlier (accessed 7 April 2020).

Ferow, A. (2019), “Childhood Grief and Loss”, European Journal of Educational Sciences, pp. 1-13.

Forrester, G., Kurth, J., Vincent, P., and Oliver, M. (2018), "Schools as community assets: an exploration of the merits of an Asset-Based Community Development (ABCD) approach", Educational Review.

Galende, N. (2015), "Death and its didactics in pre-school and primary school", Procedia-Social and Behavioral Sciences, Vol. 185, pp. 91-97.

Gentle, E., Linsley, P., and Hurley, J. (2020), “"Their story is a hard road to hoe": how art-making tackles stigma and builds well-being in young people living regionally", Journal of Public Mental Health, Vol. 19, No. 2, pp. 109- 118.

Gillam, T. (2018), "Enhancing public mental health and wellbeing through creative arts participation”, Journal of Public Mental Health, Vol. 17, No. 4, pp. 148- 156.

Glazer, H. and Marcum, D. (2011), "Expressing Grief Through Storytelling”, The Journal of Humanistic Counselling, Education and Development, Vol. 42, No. 2, pp. 131-138.

Guldin M., Li, J., Pederson, H., Obel, C., Agerbo, E., Gissler, M., Cnattingius, S., Olsen, J., and Vestergaard, M. (2015), "Incidence of Suicide Among Persons Who Had a Parent Who Died During Their Childhood: A Population-Based Cohort Study", JAMA Psychiatry, Vol. 72, pp. 1227-34. 
Jensen, B., Dür, W., and Buijis, G. (2017), "The Application of Salutogenesis in Schools”, in Mittelmark, M., Sagy, S., Eriksson, M., Bauer, G., Pelikan, J., Lindstrom, B. and Espnes, G. (eds.), The Handbook of Salutogenesis, Springer, Cham.

Kaplow, J., Saunders, J., Angold, A., and Costello, E. (2010), "Psychiatric symptoms in bereaved versus nonbereaved youth and young adults: a longitudinal epidemiological study", Journal of the American Academy of Child and Adolescent Psychiatry, Vol. 49, No. 11, pp. 1145-1154.

Kellehear, A. (2014), "Death education as a public health issue", in Stillion, J. and Attig, T. (eds.), Death, dying and bereavement: Contemporary perspectives institutions and practices, Springer, New York, NY, pp. 221-232.

Kennedy, C., Keeffe, M., Gardner, F., and Farrelly, C. (2017), "Making death, compassion and partnership 'part of life' in school communities", Pastoral Care in Education, Vol. 35, No. 2, pp. 111-123.

Kretzmann, P. and McKnight, J. (1993), Building Communities from the Inside Out: A Path Toward Finding and Mobilizing a Community's Assets, Asset-Based Community Development Institute, Evanston, IL.

Kumar, S. (2020), "Community participation in palliative care: Reflections from the ground”, Progress in Palliative Care, Vol. 28, No. 2, pp. 83-88.

LaFreniere, L., and Cain, A. (2015), "Peer Interactions of Parentally Bereaved Children and Adolescents: A Qualitative Study", OMEGA - Journal of Death and Dying, Vol. 72, No. 2, pp. 91-118.

Langford, R., Bonell, C., Jones, H., Pouliou, T., Murphy, S., Waters, E., Komro, K., Gibbs, L., Magnus, D., and Campbell, R. (2014), "The WHO Health Promoting School framework for improving the health and well-being of students and their academic achievement", Cochrane Database of Systematic Reviews 2014, No. 4, Art. No: CD008958.

McCall, B., Shallcross, L., Wilson, M., Fuller, C., and Hayward, A. (2019), "Storytelling as a research tool and intervention around public health perceptions and behaviour: a protocol for a systematic narrative review",

BMJ Open, Vol. 9, Issue 12.

McGovern, M. and Tracey, A. (2010), "A comparative examination of schools' responses to bereavement and the associated needs of the school community in Galway, West of Ireland and Derry, Northern Ireland", Pastoral Care in Education, Vol. 28, No. 3, pp. 235-252.

McLeroy, K., Bibeau, D., Steckler, A., and Glanz, K. (1988), An ecological perspective on health promotion programs. Health Education \& Behavior, Vol. 15, No. 4, pp. 351-377. 
Metzl, E. and Malissa, M. (2008), The Role of Creativity in Models of Resilience: Theoretical Exploration and Practical Applications. Journal of Creativity in Mental Health, Vol. 3, pp. 303-318.

The National Council for Palliative Care (2016), "Each Community is Prepared to Help: Community Development in End of Life Care - Guidance on Ambition Six", available at: http://endoflifecareambitions.org.uk/wpcontent/uploads/2016/09/Ambition 6 booklet.pdf (accessed 4 June 2020).

The Norfolk and Waveney Health and Care Partnership (2020), "Norfolk and Waveney STP Palliative and End of Life Care Strategy For Adults", available at: https://www.norfolkandwaveneypartnership.org.uk/publication/key-documents/73norfolk-and-waveney-palliative-and-end-of-life-care-strategy-for-adults/file (accessed 27 May 2020).

Norrish, J., Williams, P., O'Connor, M., and Robinson, J. (2013), An applied framework for Positive Education. International Journal of Wellbeing, Vol. 3.

Penny, A. and Stubbs, D. (2015), Bereavement in childhood: What do we know in 2015? National Children's Bureau, London.

Proctor, E., Silmere, H., Raghavan, R., Hovmand, P., Aarons, G., Bunger, A., Griffey R., and Hensley, M. (2011), "Outcomes for implementation research: conceptual distinctions, measurement challenges, and research agenda", Administration and policy in mental health, Vol. 38, pp. 65-76.

Rumbold, B., Gardner, F., and Nolan, I. (2011), "Spirituality and community practice", in Conway, S. (ed.), Governing Death and Loss: Empowerment, Involvement and Participation, Oxford University Press, New York, NY, pp. 139-147.

Sallnow, L. and Paul, S. (2018), "New Public Health Approaches to End-of-Life Care" in MacLeod, R. and Van den Block, L. (eds.), Textbook of Palliative Care, Springer, Cham.

Scott, S., Brett-MacLean, P., Archibald, M., and Hartling, L. (2013), "Protocol for a systematic review of the use of narrative storytelling and visual-arts- based approaches as knowledge translation tools in healthcare", Systematic Reviews, Vol. 2, No. 19.

Smith, J., Bekker, H., and Cheater, F. (2011), "Theoretical versus pragmatic design in qualitative research", Nurse Reearcher, Vol. 18, No. 2, pp. 39-51.

Stikkelbroek, Y., Bodden, D., Reitz, E., Vollebergh, W., and van Baar, A. (2016), "Mental health of adolescents before and after the death of a parent or sibling", European Child and Adolescent Psychiatry, Vol. 25, pp. 49-59.

St Nicholas Hospice Care (2020), “Life's Questions", available at: https://stnicholashospice.org.uk/support-and-information/education/lifes-questions/, (accessed 27 May 2020). 
van der Geest, I., van den Heuvel-Eibrink, M., van Vliet, L., Pluijm, S., Streng, I., Michiels, E., Pieters, R., and Darlington, A.-S. (2015), "Talking about Death with Children with Incurable Cancer: Perspectives from Parents", The Journal of Paediatrics, Vol. 167, No 6, pp. 1320-1326.

Vijay, D. and Monin, P. (2018), "Poisedness for social innovation: the genesis and propagation of community-based palliative care in Kerala (India)", M@n@gement, Vol. 21, No 4, pp. $1329-1356$.

Wegleitner, K., Heimerl, K., and Kellehear, A. (2015), Compassionate Communities. Case Studies from Britain and Europe, Routledge, London.

Winston's Wish (2020), "Facts and Figures", available at: https://www.winstonswish.org/about-us/facts-and-figures/ (accessed 4th March 2020).

\section{FURTHER READING}

Child Bereavement UK (2020), "Books and Resources", available at:

https://www.childbereavementuk.org/primary-schools-books-and-resources (access 27 May 2020). 